\title{
Nilpotent subspaces of maximal dimension in semi-simple Lie algebras
}

\author{
Jan Draisma, Hanspeter Kraft and Jochen Kuttler
}

\begin{abstract}
We show that a linear subspace of a reductive Lie algebra $\mathfrak{g}$ that consists of nilpotent elements has dimension at most $\frac{1}{2}(\operatorname{dim} \mathfrak{g}-\mathrm{rk} \mathfrak{g})$, and that any nilpotent subspace attaining this upper bound is equal to the nilradical of a Borel subalgebra of $\mathfrak{g}$. This generalizes a classical theorem of Gerstenhaber, which states this fact for the algebra of $(n \times n)$-matrices.
\end{abstract}

\section{Results and method}

A classical theorem of Gerstenhaber [Ger58] states that any vector space consisting of nilpotent $(n \times n)$-matrices has dimension at most $\left(\begin{array}{l}n \\ 2\end{array}\right)$, and that any such space attaining this maximal possible dimension is conjugate to the space of upper triangular matrices. We will prove the following generalization of this result.

Main Theorem. Let $V$ be a linear subspace of a complex semi-simple Lie algebra $\mathfrak{g}$ consisting of ad-nilpotent elements. Then $\operatorname{dim} V \leqslant \frac{1}{2}(\operatorname{dim} \mathfrak{g}-\mathrm{rk} \mathfrak{g})$ and, if equality holds, then $V$ is the nilradical of a Borel subalgebra of $\mathfrak{g}$.

The first claim of the Main Theorem is known [MR98]. We have the following easy argument. The subspaces of $\mathfrak{g}$ of a fixed dimension $m$ consisting of ad-nilpotent elements form a closed subset $Z_{m}$ of the Grassmannian $\mathrm{Gr}_{m}(\mathfrak{g})$, and $Z_{m}$ is stable under the adjoint group $G$ of $\mathfrak{g}$. If the projective variety $Z_{m}$ is not empty and if $B$ is a Borel subgroup of $G$, then $B$ stabilizes a $V \in Z_{m}$ by Borel's fixed point theorem. It is then easy to see that $V$ is contained in the Lie algebra of the unipotent radical of $B$ (see Lemma 1$)$.

The difficult part of the theorem is the statement that the subspaces consisting of ad-nilpotent elements of maximal dimension $d:=\frac{1}{2}(\operatorname{dim} \mathfrak{g}-\mathrm{rk} \mathfrak{g})$ are nilpotent subalgebras and are therefore all conjugate. The maximality assumption is essential here: there exist subspaces consisting of ad-nilpotent elements that are not contained in nilpotent subalgebras (see Examples 1-A.2 below). The classification of these 'nilpotent subspaces' is widely open, even in the case of $(n \times n)$-matrices. For some partial results see [Ger59a, Ger59b, Ger62, Fas97, CRT98, MOR91].

Gerstenhaber proves his theorem under the mild assumption that the ground field $K$ has at least $n$ elements. We will prove a version of the Main Theorem which holds in arbitrary characteristic (see Theorem 1), but still assumes that $K$ is algebraically closed. To formulate this more technical result, let $G$ be a connected reductive algebraic group over $K$ and denote its Lie algebra by $\mathfrak{g}$. Fix a Borel subgroup $B$ of $G$ and a maximal torus $T$ of $G$ contained in $B$, and denote by $X(T)$ the character group of $T$. Let $W:=N_{G}(T) / T$ be the Weyl group of $(G, T)$. Denote by $\Phi \subset X(T)$ the root system of $(G, T)$, by $\Delta=\left\{\alpha_{1}, \alpha_{2}, \ldots, \alpha_{r}\right\} \subset \Phi$ the simple roots relative to $B$, and by $\Phi^{ \pm}$ 


\section{MAXimal NiLPotent SUBSPACES}

the positive and negative roots, respectively. Then we have the usual decompositions

$$
\mathfrak{g}=\mathfrak{n}^{-} \oplus \mathfrak{h} \oplus \mathfrak{n}=\mathfrak{n}^{-} \oplus \mathfrak{b}, \quad \mathfrak{n}:=\bigoplus_{\alpha \in \Phi^{+}} \mathfrak{g}_{\alpha}, \quad \text { and } \quad \mathfrak{n}^{-}:=\bigoplus_{\alpha \in \Phi^{+}} \mathfrak{g}_{-\alpha},
$$

where $\mathfrak{g}_{\alpha}$ is the root space of weight $\alpha, \mathfrak{h}$ is the Lie algebra of $T, \mathfrak{n}$ is the Lie algebra of the unipotent radical $U$ of $B, \mathfrak{b}=\mathfrak{h} \oplus \mathfrak{n}$ is the Lie algebra of $B$, and $\mathfrak{n}^{-}$is the Lie algebra of the unipotent radical of the opposite Borel subgroup $B^{-}$. As a general reference for algebraic group theory we use Borel's book [Bor91].

We will replace the term 'ad-nilpotent' in the Main Theorem by 'nilpotent', as in Gerstenhaber's statement. Recall that an element $x$ of $\mathfrak{g}$ is said to be nilpotent if it acts locally nilpotently on the coordinate ring $K[G]$ of $G$. As every finite-dimensional rational representation of $G$ is a submodule of a direct sum of copies of $K[G]$, this is equivalent to the condition that $x$ acts nilpotently on all finite-dimensional rational $G$-modules. Moreover, since homomorphisms of algebraic groups preserve the Jordan decomposition, this is equivalent to the condition that $x$ acts nilpotently on some finitedimensional rational $G$-module that is faithful for $\mathfrak{g}$. In particular, if the center of $\mathfrak{g}$ is trivial, then $x$ is nilpotent if and only if it is ad-nilpotent. This holds, for example, if $G$ is semi-simple and $\operatorname{char} K=0$.

In what follows, a subspace of $\mathfrak{g}$ consisting of nilpotent elements will be called a nilpotent subspace. Moreover, we reserve the term nilpotent subalgebra for a subalgebra of $\mathfrak{g}$ that is $G$-conjugate to a subalgebra of $\mathfrak{n}$, even though there are other subalgebras which are nilpotent as abstract Lie algebras. A maximal nilpotent subalgebra is then a conjugate of $\mathfrak{n}$. Similarly, by a Borel subalgebra we mean the Lie algebra of a Borel subgroup.

The natural generalization of the Main Theorem in this context would be the following.

Desired Generalization. Any subspace $V$ of $\mathfrak{g}$ consisting of nilpotent elements has dimension at most $\operatorname{dim} \mathfrak{n}$ and if equality holds, then $V$ is a maximal nilpotent subalgebra of $G$.

However, the statement is false in this generality. A first class of counter-examples stems from the following subtlety. Suppose that $G^{\prime}$ is another connected, reductive algebraic group and that $\varphi: G^{\prime} \rightarrow G$ is an isogeny, i.e. a surjective homomorphism with finite kernel. If $\mathrm{d}_{e} \varphi: \mathfrak{g}^{\prime} \stackrel{\sim}{\longrightarrow} \mathfrak{g}$ is an isomorphism, then the generalization above holds for $G$ if and only if it holds for $G^{\prime}$. In characteristic zero this is always the case, but in positive characteristic $\operatorname{ker} \mathrm{d}_{e} \varphi$ may be non-trivial, and the following example shows that this leads to interesting counter-examples.

Example 1. Suppose that char $K=p$ and consider $G=\operatorname{PSL}_{p}$ and $G^{\prime}=\operatorname{SL}_{p}$ with $\varphi: G^{\prime} \rightarrow G$ corresponding to the adjoint representation. Let $x$ be a matrix in $\mathfrak{g}^{\prime}$ of the form

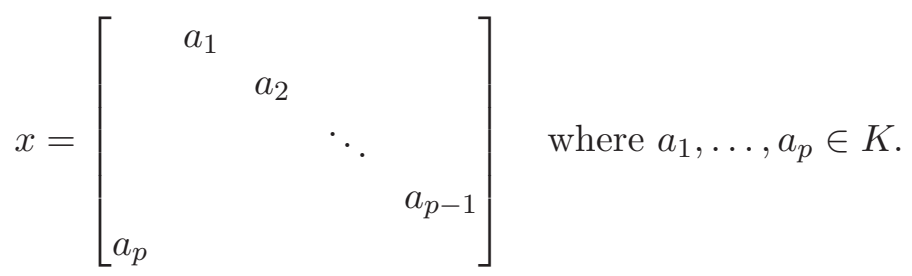

Then the characteristic polynomial of $x$ is $t^{p}-a$, where $a:=a_{1} a_{2} \cdots a_{p}$. Hence, the Jordan decomposition of $x$ is $x=b I+(x-b I)$ where $b$ denotes the unique $p$ th root of $a$ in $K$. As the semi-simple part $b I$ is in the kernel of the adjoint representation, $\mathrm{d}_{e} \varphi(x)$ is nilpotent. We conclude that the space $V$ consisting of all $x$ as above is a $p$-dimensional nilpotent subspace of $\mathfrak{g}$. Moreover, one readily checks that $V$ generates a subalgebra of $\mathfrak{g}$ strictly containing $\mathfrak{n}$ and therefore $V$ is certainly not conjugate to a subspace of $\mathfrak{n}$; alternatively, this follows from Lemma A.4 in Appendix A, $\S$ A.3. For $p>3$ we have $\operatorname{dim} V=p<\operatorname{dim} \mathfrak{n}$, so that $V$ does not satisfy the maximality assumption; but for $p=2$ and 3 we do obtain counter-examples to the desired generalization. 


\section{J. Draisma, H. Kraft and J. Kuttler}

Another exception to the generalization above is the following.

Example 2. Suppose that $G$ is of type $G_{2}$ and that char $K=2$. Then it is known that the zero weight space $M_{0}$ in the seven-dimensional Weyl module $M$ of highest weight $\omega_{1}$ is a trivial $G$-submodule and that $\mathfrak{g}$ acts faithfully on the quotient $M / M_{0}$. (See, for example, [Kal73, p. 91, Table 3], where the 0 in the second row means that zero is not a weight in the irreducible module of highest weight $\omega_{1}$. For more about $G_{2}$ and about representation theory of reductive groups in positive characteristic we refer to [SV00] and [Jan03].) It is now readily verified in the weight system of $M / M_{0}$ that the subspace

$$
V:=\mathfrak{g}_{-2 \alpha_{1}-\alpha_{2}} \oplus \bigoplus_{\alpha \in \Phi^{+} \backslash\left\{2 \alpha_{1}+\alpha_{2}\right\}} \mathfrak{g}_{\alpha}
$$

acts nilpotently on $M / M_{0}$. Thus, $V$ is a six-dimensional $T$-stable nilpotent subspace of $\mathfrak{g}$. Although $V$ is a subalgebra (in fact, it is the intersection of $\mathfrak{g}$ with the unipotent radical of a Borel subgroup of $\mathrm{SL}\left(M / M_{0}\right)$ ) it is not conjugate to $\mathfrak{n}$, as one can see using Lemma A.4 in Appendix A, $\S$ A.3.

Surprisingly, the desired generalization of the Main Theorem becomes true if we avoid $\mathrm{PSL}_{2}$ and $G_{2}$ in characteristic two, and $\mathrm{PSL}_{3}$ in characteristic three. More precisely, we have to prevent their occurrence as subgroups of $G$ in the following way. For a subsystem $\Psi$ of $\Phi$, by which we mean the intersection of $\Phi$ with a subspace of $X(T)_{\mathbb{R}}:=X(T) \otimes_{\mathbb{Z}} \mathbb{R}$, we write $G_{\Psi}$ for the centralizer of $\left(\bigcap_{\alpha \in \Psi} \operatorname{ker} \alpha\right)^{0}$. The latter group is a subtorus of $T$ whose codimension is the rank of $\Psi$. The Levi subgroup $G_{\Psi}$ is a connected reductive algebraic group with root system $\Psi$, so its derived subgroup $\left(G_{\Psi}, G_{\Psi}\right)$ is semi-simple. For $\Psi=\{-\alpha, \alpha\}$ we write $G_{\alpha}$ for $G_{\Psi}$.

In the rest of this paper we will repeatedly refer to the following conditions.

Condition 1. If char $K=2$, then $\left(G_{\alpha}, G_{\alpha}\right) \cong \mathrm{SL}_{2}$ for all $\alpha \in \Phi$.

Condition 2. If char $K=3$, then $\left(G_{\Psi}, G_{\Psi}\right) \cong \mathrm{SL}_{3}$ for all $\Psi \subseteq \Phi$ of type $A_{2}$.

Condition 3. If char $K=2$, then $\Phi$ has no simple component of type $G_{2}$.

In Appendix A, $\S$ A.2 we show that Conditions 1 and 2 are fulfilled if $G$ is almost simply connected, that is, if every simple component of $(G, G)$ is simply connected (see Lemma A.3). We also show that Condition 1 implies $\left[\mathfrak{g}_{\alpha}, \mathfrak{g}_{-\alpha}\right] \neq 0$ for all roots $\alpha$ (see Lemma A.2). This is the property that we will need most frequently.

Theorem 1. Suppose that the connected reductive algebraic K-group $G$ satisfies Conditions 1-3. Let $V$ be a nilpotent subspace of its Lie algebra $\mathfrak{g}$ and denote by $\mathfrak{n}$ the Lie algebra of a maximal unipotent subgroup of $G$. Then $\operatorname{dim} V \leqslant \operatorname{dim} \mathfrak{n}$ and if equality holds, then $V$ is conjugate to $\mathfrak{n}$.

By our remarks on nilpotency above, the Main Theorem is an immediate consequence of Theorem 1.

Remark. Let $G$ be an arbitrary connected algebraic group. If $R_{u}(G)$ is its unipotent radical, then any maximal-dimensional nilpotent subspace $V$ of $\operatorname{Lie}(G)$ contains $\mathfrak{u}:=\operatorname{Lie} R_{u}(G)$. Indeed, $x \in V$ implies that $x$ is contained in the Lie algebra of a maximal unipotent subgroup of $G$, whose Lie algebra clearly contains $\mathfrak{u}$. Hence $x+\mathfrak{u}$ is nilpotent. It immediately follows that $V / \mathfrak{u}$ is maximal in $\mathfrak{g} / \mathfrak{u}$, and thus $V$ is a maximal nilpotent subalgebra, assuming that char $K \neq 2,3$ or $G / R_{u}(G)$ satisfies our additional Conditions 1-3.

We conclude this section by giving a short outline of our proof of Theorem 1 . The argument given above for complex semi-simple Lie algebras still serves to show that $\operatorname{dim} V \leqslant \operatorname{dim} \mathfrak{n}=: d$. Concerning the second part, we first prove it for $T$-stable nilpotent subspaces of dimension $d$ (Proposition 1 ). 


\section{MAXimal NiLPotent SUBSPACES}

Then we show that a nilpotent subspace $V$ of dimension $d$ contains a semi-regular nilpotent element $x$ of $\mathfrak{g}$, i.e. an element $x$ that lies in a unique Borel subalgebra (see Appendix A, $\S$ A.1 for details on semi-regularity). By the conjugacy of Borel subgroups we may assume that $x$ lies in $\mathfrak{n}$, so that, if $V$ is to be conjugate to $\mathfrak{n}$, semi-regularity of $x$ forces $V$ to be equal to $\mathfrak{n}$.

Now we use a one-parameter subgroup $\mu: K^{*} \rightarrow T$ having $\bigoplus_{i} \mathfrak{g}_{\alpha_{i}}$ as a weight space with positive weight $n_{0}$, so that $\mu$ has positive weights $n_{0}, 2 n_{0}, \ldots$ on $\mathfrak{n}$, negative weights $-n_{0},-2 n_{0}, \ldots$ on $\mathfrak{n}^{-}$ and weight 0 on $\mathfrak{h}$. If the limit in $\operatorname{Gr}_{d}(\mathfrak{g})$ of $\mu(t) \cdot V$ for $t \rightarrow 0$ is equal to $\mathfrak{n}$, then it is easy to see that no element of $V$ has a non-zero component in $\mathfrak{n}^{-} \oplus \mathfrak{h}$, so that $V$ is equal to $\mathfrak{n}$, too. Therefore, it suffices to prove $V=\mathfrak{n}$ under the additional assumption that $V$ is equal to this limit, i.e. that $V$ is $\mu$-stable.

This implies that the component $x_{0}$ of $x$ of weight $n_{0}$ lies in $V$, and it follows that this component $x_{0}$ is a sum of simple root vectors which, by semi-regularity, is supported in all simple roots. Now comes the core of our proof, where we show, by induction on the semi-simple rank of $G$, that $V$ contains, along with $x$, all simple root spaces $\mathfrak{g}_{\alpha_{i}}$.

Finally, we choose a regular one-parameter subgroup $\lambda: K^{*} \rightarrow T$, i.e. one whose weight spaces in $\mathfrak{g}$ are precisely the root spaces. Then $V_{0}:=\lim _{t \rightarrow 0} \lambda(t) \cdot V$ and $V_{\infty}:=\lim _{t \rightarrow \infty} \lambda(t) \cdot V$ are both $T$-stable $d$-dimensional nilpotent subspaces of $\mathfrak{g}$ containing all simple root spaces. Since we already know the theorem for $T$-stable nilpotent subspaces, it follows that $V_{0}=\mathfrak{n}=V_{\infty}$. This implies that $\lambda$ stabilizes $V$, because an orbit of $K^{*}$ on $\operatorname{Gr}_{d}(\mathfrak{g})$ has two distinct 'end points' unless it is a point. Hence, $V$ is $T$-stable, and therefore $V=\mathfrak{n}$.

The rest of this paper is organized as follows. In $\S 2$ we prove the dimension bound for nilpotent subspaces and we deal with $T$-stable nilpotent subspaces attaining this bound. Section 3 fills in all details in the proof above, and in Appendix A we collect some technical lemmas ensuring that our method works under Conditions $1-3$ on $G$ above.

\section{Dimension bound and $T$-stable nilpotent subspaces}

Retaining the notation of $\S 1$, we first prove the dimension bound in Theorem 1 . Let $\mathcal{N}_{\mathfrak{g}}$ be the nilpotent cone in $\mathfrak{g}$,

$$
\mathcal{N}_{\mathfrak{g}}:=G \mathfrak{n}=\{g x \mid g \in G, x \in \mathfrak{n}\}
$$

where we write $g x$ (rather than $g x g^{-1}$ ) for the adjoint action of $g$ on $x$. For any natural number $m$, define

$$
Z_{m}:=\left\{V \in \operatorname{Gr}_{m}(\mathfrak{g}) \mid V \subseteq \mathcal{N}_{\mathfrak{g}}\right\} .
$$

Clearly, $Z_{m}$ is a closed $G$-stable subset of the Grassmannian $\operatorname{Gr}_{m}(\mathfrak{g})$.

Lemma 1. Under Condition 1 the closed $G$-orbits in $Z_{m}$ consist of nilpotent subalgebras. In particular, if $Z_{m}$ is non-empty then $m \leqslant \operatorname{dim} \mathfrak{n}$, and if equality holds then $Z_{m}$ contains a unique closed orbit, namely the orbit of $\mathfrak{n}$.

Proof. By Borel's fixed point theorem [Bor91, III.10.4] any closed $G$-orbit in $Z_{m}$ contains a point $V$ fixed by $B$. In particular $V$ is $T$-stable, and as $V$ cannot intersect $\mathfrak{h}$, it is a sum of root spaces. By Condition 1 and Lemma A.2 we have $\left[\mathfrak{g}_{\alpha}, \mathfrak{g}_{-\alpha}\right] \neq 0$ for all $\alpha \in \Phi_{+}$, so that the $B$-module generated by a negative root space $\mathfrak{g}_{-\alpha}$ contains non-zero elements of $\mathfrak{h}$. Hence $V$ is contained in $\mathfrak{n}$. However, then it is a $\mathfrak{b}$-ideal in $\mathfrak{n}$, and hence a nilpotent subalgebra of $\mathfrak{g}$. The last two statements of the lemma are now immediate.

From now on, we will be interested only in nilpotent subspaces of the maximal possible dimension

$d:=\operatorname{dim} \mathfrak{n}=\frac{1}{2}(\operatorname{dim} G-\operatorname{rk} G)=\left|\Phi^{+}\right|$. By slight abuse of language, we will call these, and only these, 


\section{J. Draisma, H. Kraft and J. Kuttler}
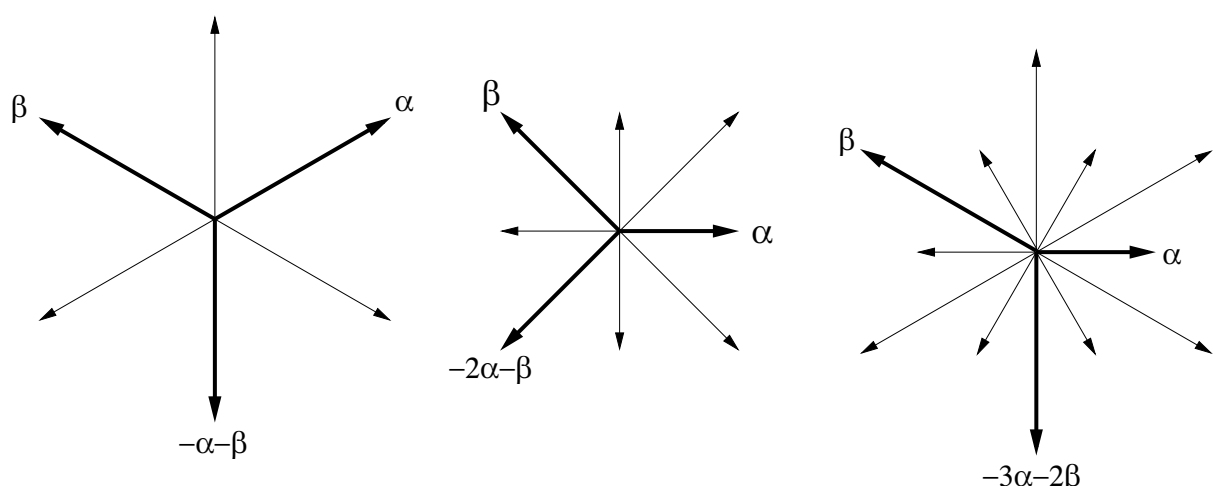

Figure 1. Supports of cyclic elements in $A_{2}, C_{2}$, and $G_{2}$.

the maximal nilpotent subspaces, although they are by no means the only nilpotent subspaces that are maximal with respect to inclusion (see Examples A.1 and A.2 in Appendix A.3).

We now proceed to prove Theorem 1 for $T$-stable nilpotent subspaces. For $G=\mathrm{SL}_{n}$ it is easy to see that any $T$-stable nilpotent subspace $V$ is conjugate to a subspace of $\mathfrak{n}$ [FP96]. Even in characteristic zero, this statement is not true for general $G$ (see Example A.1 in Appendix A.3), but it holds for nilpotent subspaces of maximal dimension.

Proposition 1. Let $\Psi$ be a subset of $\Phi$ of size $d=\left|\Phi^{+}\right|$, and suppose that $V_{\Psi}:=\bigoplus_{\gamma \in \Psi} \mathfrak{g}_{\gamma}$ is a nilpotent subspace.

(i) If Condition 1 holds, then

$$
|\Psi \cap\{\alpha,-\alpha\}|=1 \text { for all } \alpha \in \Phi .
$$

(ii) Suppose that Conditions 1-3 are fulfilled. Then $\Psi$ is $W$-conjugate to $\Phi^{+}$.

Proof. (i) For all $\alpha \in \Phi$, the subspace $V_{\Psi} \cap\left(\mathfrak{g}_{\alpha} \oplus \mathfrak{g}_{-\alpha}\right)$ of $\operatorname{Lie}\left(G_{\alpha}\right)$ is $T$-stable and nilpotent. By Lemma 1 , it is at most one-dimensional. Hence $\Psi$ cannot contain both a root and its negative. Together with $|\Psi|=\left|\Phi^{+}\right|$this implies the claim.

(ii) It suffices now to prove that $\Psi$ is a closed subset of $\Phi$, i.e. that $\alpha, \beta \in \Psi$ and $\alpha+\beta \in \Phi$ together imply $\alpha+\beta \in \Psi$. Indeed, in that case $\Psi$ is the set of positive roots relative to some choice of simple roots [Bou68, ch. VI, No. 1.7], hence $W$-conjugate to $\Phi^{+}$.

To show that $\Psi$ is indeed closed, let $\alpha, \beta \in \Psi$ be such that $\alpha+\beta$ is a root. Then $\alpha, \beta$ lie in a simple subsystem $\Sigma$ of $\Phi$ of rank 2 , and

$$
V_{\Psi} \cap \operatorname{Lie}\left(\left(G_{\Sigma}, G_{\Sigma}\right)\right)=V_{\Psi \cap \Sigma}
$$

is a $T$-stable nilpotent subspace. It is maximal, because $\Psi \cap \Sigma$ satisfies the condition (i) with $\Phi$ replaced by $\Sigma$. Hence, to prove that $\Psi$ is closed we may as well assume that $G=\left(G_{\Sigma}, G_{\Sigma}\right)$, and is simple of rank 2.

For each of the possible Cartan types $A_{2}, C_{2}$, and $G_{2}$ of $\Phi$ it is easily verified that if $\Psi$ satisfies (i) and is not conjugate to $\Phi^{+}$, then $\Psi$ contains a subset conjugate to $\Delta \cup\left\{-\alpha_{\max }\right\}$, where $\alpha_{\max }$ denotes the highest root (see Figure 1). Hence we may assume that $\Delta \cup\left\{-\alpha_{\max }\right\} \subset \Psi$. However, the following lemma asserts that this is impossible.

Lemma 2. Suppose that $G$ is simple of rank 2 and that Conditions 1-3 hold. For each $\gamma \in \Psi:=$ $\Delta \cup\left\{-\alpha_{\max }\right\}$ let $x_{\gamma}$ be a non-zero element of $\mathfrak{g}_{\gamma}$. Then $\sum_{\gamma \in \Psi} x_{\gamma}$ is not nilpotent. 


\section{MaXimal NiLPOTENT SUBSPACES}

We give a case-by-case proof of this lemma in Appendix A, $\S$ A.4. It is here that $G_{2}$ has to be excluded in characteristic two (Condition 3), and that $\mathrm{PSL}_{3}$ has to be excluded in characteristic three (Condition 2). In fact, $\mathrm{PSp}_{4}$ in characteristic two must also be excluded, but Condition 1 already does so.

We point out here that in characteristic zero, the following stronger statement due to Kostant is true.

Lemma 3 [Kos59, Lemma 6.3 and Corollary 6.4]. Suppose that char $K=0$ and $\mathfrak{g}$ is simple. For each $\gamma \in \Psi:=\Delta \cup\left\{-\alpha_{\max }\right\}$ let $x_{\gamma}$ be a non-zero element of $\mathfrak{g}_{\gamma}$. Then $\sum_{\gamma \in \Psi} x_{\gamma}$ is regular semi-simple.

Kostant calls such elements cyclic (cf. Example 1). In fact, he shows that they form one projective orbit under $T$, and then that one particular cyclic element is regular semi-simple.

\section{Proof of Theorem 1}

To fill in the details of the proof outlined in $\S 1$, we assume the notation introduced there, as well as the additional notation of $\S 2$.

\subsection{Maximal nilpotent subspaces contain semi-regular elements}

We call a nilpotent element $x \in \mathfrak{g}$ semi-regular if it is contained in the Lie algebra of a unique Borel subgroup of $G$. We prove in Appendix A (Lemma A.1) that the semi-regular nilpotent elements form an open dense subset of the nilpotent cone $\mathcal{N}_{\mathfrak{g}}$ and that an element $x=\sum_{\alpha \in \Phi^{+}} x_{\alpha}$ of $\mathfrak{n}$ with $x_{\alpha} \in \mathfrak{g}_{\alpha}$ is semi-regular if and only if $x_{\alpha_{i}}$ is non-zero for all simple roots $\alpha_{i}$.

Proposition 2. Assume Condition 1 and let $V$ be any nilpotent subspace of $\mathfrak{g}$.

(i) If $V$ is maximal, then it contains a semi-regular nilpotent element.

If char $K=0$, then we have in addition:

(ii) $V$ is isotropic with respect to the Killing form $\kappa$, i.e. $V^{\perp} \supset V$;

(iii) if $V$ is maximal, then $V^{\perp} \cap \mathfrak{n}=V \cap \mathfrak{n}$.

Part of this proposition is also proved in [MR98]. The last two statements can be used to give a slightly shorter proof of our results in characteristic zero. They will not be used in what follows.

Proof. (i) That $V \in Z_{d}$ does not contain a semi-regular nilpotent element is equivalent to the condition that $V \subset C$ for the closed $G$-stable cone $C \subset \mathcal{N}_{g}$ consisting of non-semi-regular elements. It follows that this is a closed and $G$-invariant condition on $Z_{d}$. So if any $V \in Z_{d}$ satisfies this condition, then so does a closed $G$-orbit in $Z_{d}$. However, by Lemma 1 , this would imply that $\mathfrak{n}$ does not contain a semi-regular element, a contradiction.

(ii) Any nilpotent element of $\mathfrak{g}$ is isotropic. Hence, for $A, B \in V$ we have $0=\kappa(A+B, A+B)=$ $\kappa(A, A)+2 \kappa(A, B)+\kappa(B, B)=2 \kappa(A, B)$.

(iii) If $K$ has characteristic zero, then $\kappa$ is non-degenerate. By (ii) we get $V^{\perp} \cap \mathfrak{n} \supset V \cap \mathfrak{n}$. On the other hand, we have $\mathfrak{b}^{\perp}=\mathfrak{n}$, so that $V^{\perp} \cap \mathfrak{n}=(V+\mathfrak{b})^{\perp}$. Now $\operatorname{dim}(V+\mathfrak{b})=\operatorname{dim} V+\operatorname{dim} \mathfrak{b}-$ $\operatorname{dim}(V \cap \mathfrak{b})=\operatorname{dim} \mathfrak{g}-\operatorname{dim}(V \cap \mathfrak{n})$, where we have used $V \cap \mathfrak{b}=V \cap \mathfrak{n} . T$ Thus $\operatorname{dim}\left(V^{\perp} \cap \mathfrak{n}\right)=\operatorname{dim}(V \cap \mathfrak{n})$ as required. 


\section{J. Draisma, H. Kraft and J. Kuttler}

\subsection{Actions of one-parameter subgroups}

We collect some well-known facts about one-parameter subgroups of $G$ and their actions on subspaces of $\mathfrak{g}$. Let $\lambda: K^{*} \rightarrow G$ be a one-parameter subgroup of $G$ (1-PSG). If $V$ is a maximal nilpotent subspace of $\mathfrak{g}$ then so is $\lambda(t) \cdot V:=\{\lambda(t) v \mid v \in V\}$ for all $t \in K^{*}$. Viewing $\lambda(t) \cdot V$ as an element of $\mathrm{Gr}_{d}(\mathfrak{g})$, the two limits

$$
V_{0}:=\lim _{t \rightarrow 0} \lambda(t) \cdot V \quad \text { and } \quad V_{\infty}:=\lim _{t \rightarrow \infty} \lambda(t) \cdot V
$$

exist, and both are $\lambda$-stable maximal nilpotent subspaces. Moreover, $V_{0} \neq V_{\infty}$ unless $V$ is already $\lambda$-stable, because a one-dimensional $K^{*}$-orbit in a normal projective variety has two distinct 'end points'. This is clear for (linear) actions on projective spaces, the only case we will need here, and holds for projective normal varieties since those admit an equivariant embedding into a projective space by a famous theorem of Sumihiro [Sum74, Sum75], cf. [KKL89].

If $G$ is semi-simple of adjoint type, then the simple roots form a basis of $X(T)$. Therefore, there exist 1-PSGs $\lambda_{1}, \ldots, \lambda_{r}: K^{*} \rightarrow T$ such that $\left\langle\alpha_{j}, \lambda_{k}\right\rangle=\delta_{j k}$, where $\langle$,$\rangle denotes the usual pairing$ between $X(T)$ and the group $Y(T):=\operatorname{Hom}\left(K^{*}, T\right)$ of 1-PSGs of $T$. For general semi-simple $G$, the simple roots span a sublattice of finite index in $X(T)$, so that we can still find a positive integer $n_{0}$ and 1-PSGs $\lambda_{1}, \ldots, \lambda_{r}$ such that $\left\langle\alpha_{j}, \lambda_{k}\right\rangle=n_{0} \delta_{j k}$. Finally, if $G$ is an arbitrary reductive group, then the last remark applies to $(G, G)$, and such $\lambda_{i}$ exist in this case as well.

We call a 1-PSG $\lambda: K^{*} \rightarrow T$ regular (with respect to $\mathfrak{g}$ ) if it has the same eigenspaces in $\mathfrak{g}$ as $T$. It is well known that regular 1-PSGs exist (see [Hum75]).

Lemma 4. Let $\mu: K^{*} \rightarrow T$ be a 1-PSG and let $V$ be a $\mu$-stable maximal nilpotent subspace of $\mathfrak{g}$. Denote by $V=\bigoplus_{j} V(j)$ the decomposition of $V$ into $\mu$-weight spaces.

(i) If $\mu$ is regular, then $V$ is $T$-stable.

(ii) Assume Condition 1. Let $G^{\mu}$ denote the Levi subgroup of $G$ fixed by $\mu$ and set $\mathfrak{g}^{\mu}:=\operatorname{Lie}\left(G^{\mu}\right)$. Then $V^{\mu}:=V \cap \mathfrak{g}^{\mu}$ is a maximal nilpotent subspace of $\mathfrak{g}^{\mu}$.

Let $\lambda: K^{*} \rightarrow T$ be another 1-PSG and put $V_{0}:=\lim _{t \rightarrow 0} \lambda(t) \cdot V$ and $V_{\infty}:=\lim _{t \rightarrow \infty} \lambda(t) \cdot V$.

(iii) $V_{0}, V_{\infty}$ are $\mu$-stable maximal nilpotent subspaces. In the decomposition $V_{0}=\bigoplus_{j} V_{0}(j)$ into $\mu$-weight spaces we have $V_{0}(j)=\lim _{t \rightarrow 0} \lambda(t) \cdot V(j)$, and similarly for $V_{\infty}$.

(iv) If $x \in V$ is a $\lambda$-weight vector then $x \in V_{0}$ and $x \in V_{\infty}$.

Proof. The proofs of statements (i), (ii), and (iii) are straightforward. As for (ii), we see that $V^{\mu}$ is a nilpotent subspace of $\mathfrak{g}^{\mu}$, so that it suffices to prove that $V^{\mu}$ has the maximal dimension $\operatorname{dim} \mathfrak{n} \cap \mathfrak{g}^{\mu}$. To this end, let $\lambda: K^{*} \rightarrow T$ be a regular 1-PSG, so that the $\operatorname{limit}_{0}:=\lim _{t \rightarrow 0} \lambda(t) \cdot V$ is a $T$-stable maximal nilpotent subspace of $\mathfrak{g}$. By Proposition $1(\mathrm{i})$ we see that $V_{0}$ contains either $\mathfrak{g}_{\alpha}$ or $\mathfrak{g}_{-\alpha}$ for every root $\alpha$. We conclude that $V_{0} \cap \mathfrak{g}^{\mu}$ is a maximal nilpotent subspace of $\mathfrak{g}^{\mu}$. However, by (iii) $\operatorname{dim} V_{0} \cap \mathfrak{g}^{\mu}=\operatorname{dim} V \cap \mathfrak{g}^{\mu}$, and we are done.

\subsection{Reduction to graded maximal nilpotent subspaces}

In all that follows we assume Condition 1 without further mention. Let $V$ be a maximal nilpotent subspace of $\mathfrak{g}$. By Proposition 2, $V$ contains a semi-regular element $x$, and by the conjugacy of Borel subgroups we may assume that $x$ lies in $\mathfrak{n}$. In this setting, we have to prove that $V$ is equal to $\mathfrak{n}$. Set $\mu:=\lambda_{1}+\lambda_{2}+\cdots+\lambda_{r}$, where the $\lambda_{i}$ are as above, i.e. $\left\langle\alpha_{j}, \lambda_{k}\right\rangle=n_{0} \delta_{j k}$. Then $\mu$ has weight $n_{0} \operatorname{ht}(\alpha)$ on $\mathfrak{g}_{\alpha}$, where $\mathrm{ht}(\alpha)$ denotes the height of the root $\alpha$ relative to the chosen set of simple roots.

Lemma 5. If $V_{0}:=\lim _{t \rightarrow 0} \mu(t) \cdot V$ is equal to $\mathfrak{n}$, then so is $V$. In other words, $\mathfrak{n}$ is a source of the $\mu$-action on $\operatorname{Gr}_{d}(\mathfrak{g})$. 


\section{MAXimal NiLPotent SUBSPACES}

Proof. Let $v$ be a non-zero element of $V$ and let $v_{\min }$ be the non-zero component of $v$ of lowest $\mu$-weight. Then $\lim _{t \rightarrow 0} \mu(t) \cdot K v=K v_{\text {min }} \subset V_{0}$. Hence, if $V_{0}=\mathfrak{n}$ then $v_{\text {min }} \in \mathfrak{n}$ for all non-zero $v \in V$, and therefore $V \subset \mathfrak{n}$. Now the lemma follows by maximality of $V$.

We conclude that it suffices to prove $V=\mathfrak{n}$ under the additional assumption that $V=V_{0}$ is $\mu$-stable. From now on we suppose that this is the case, and decompose $V=\bigoplus_{j} V(j)$ and $\mathfrak{g}=\bigoplus_{j} \mathfrak{g}(j)$ into $\mu$-weight spaces. Moreover, as the component of $x$ in $V\left(n_{0}\right)$ is again semiregular, we may also assume that $x=\sum_{i=1}^{r} x_{i}$ where every $x_{i} \in \mathfrak{g}_{\alpha_{i}}$ is non-zero.

Lemma 6. For all $j \neq 0$ we have $\operatorname{dim} V(j)+\operatorname{dim} V(-j)=\operatorname{dim} \mathfrak{g}(j)$.

Proof. Let $\lambda: K^{*} \rightarrow T$ be a regular 1-PSG. By Lemma 4(i), $V_{0}:=\lim _{t \rightarrow 0} \lambda(t) \cdot V$ is a $T$-stable maximal nilpotent subspace of $\mathfrak{g}$. By Proposition $1(\mathrm{i})$, it satisfies $\operatorname{dim} V_{0}(j)+\operatorname{dim} V_{0}(-j)=\operatorname{dim} \mathfrak{g}(j)$ for all $j \neq 0$. The claim now follows from Lemma 4(iii).

\subsection{Conclusion of the proof}

Under these conditions on $V$ and $x$ we are ready for the main induction argument in our proof. We now assume Conditions 2 and 3 in addition to Condition 1.

Lemma 7. Along with $x$, the $\mu$-stable maximal nilpotent space $V$ contains all simple root spaces $\mathfrak{g}_{\alpha_{i}}$.

Proof. We proceed by induction on the semi-simple rank $r$ of $G$. For $r=1$ the statement is trivial. Assume, therefore, that $r>1$ and that the lemma holds for all groups of rank $r-1$ satisfying Conditions 1-3. In particular, it then holds for rank $r-1$ Levi subgroups of $G$.

Recall the 1-PSGs $\lambda_{1}, \ldots, \lambda_{r}$ 'dual' to the simple roots, let $L_{k}:=G^{\lambda_{k}}$ be the corresponding Levi subgroups of $G$, and write $\mathfrak{l}_{k}:=\operatorname{Lie}\left(L_{k}\right)$. Now fix a $k \in\{1, \ldots, r\}$. Clearly, $\bar{x}:=\sum_{i \neq k} x_{i}=$ $\lim _{t \rightarrow 0} \lambda_{k}(t) x$ lies in $V_{0}:=\lim _{t \rightarrow 0} \lambda_{k}(t) \cdot V$. By construction, $V_{0}$ is a $\lambda_{k}$-stable maximal nilpotent subspace of $\mathfrak{g}$ and consequently $V_{0}^{\lambda_{k}}=V_{0} \cap \mathfrak{l}_{k}$ is a maximal nilpotent subspace of $\mathfrak{l}_{k}$ (Lemma 4(ii)) containing $\bar{x}$. Moreover, $V_{0}^{\lambda_{k}}$ is $\mu$-stable (Lemma $4($ iii)), and hence still graded by root height. We may therefore apply the induction hypothesis and find that $V_{0}^{\lambda_{k}}\left(n_{0}\right)=\bigoplus_{i \neq k} \mathfrak{g}_{\alpha_{i}}$. In particular, $\operatorname{dim} V\left(n_{0}\right) \geqslant r-1$ and hence $\operatorname{dim} V\left(-n_{0}\right) \leqslant 1$ by Lemma 6 .

Now if $V\left(-n_{0}\right)$ does not contain a semi-regular element, then we may choose the $k$ above such that $V\left(-n_{0}\right)$ is contained in $\bigoplus_{i \neq k} \mathfrak{g}_{-\alpha_{i}}$, so that $V\left(-n_{0}\right)=V_{0}\left(-n_{0}\right)=V_{0}^{\lambda_{k}}\left(-n_{0}\right)=0$, and we are done, again by Lemma 6.

Suppose, therefore, that $V\left(-n_{0}\right)$ does contain a semi-regular element $x^{\prime}$. Then the same argument as above (with $\mu$ replaced by $\mu^{-1}$ ) shows that $\operatorname{dim} V\left(-n_{0}\right) \geqslant r-1$. Together with $\operatorname{dim} V\left(-n_{0}\right) \leqslant 1$, this implies $\operatorname{dim} V\left(-n_{0}\right)=1$ and $r=2$. If $\Phi$ is of type $A_{1} \oplus A_{1}$, then the projection of $K x^{\prime} \oplus K x$ onto either of the two copies of $A_{1}$ is two-dimensional and thus not nilpotent by Lemma 1 . Therefore, this cannot occur and we may assume that $\Phi$ is simple of rank 2 . One readily verifies, then, that the $\mu$-weight spaces $\mathfrak{g}(j)$ with $j \neq 0, \pm n_{0}$ are precisely the root spaces $\mathfrak{g}_{\alpha}$ with $\alpha$ non-simple. Now by Lemma $6, V$ contains one of the spaces $K x+\mathfrak{g}_{-\alpha_{\max }}$ or $K x^{\prime}+\mathfrak{g}_{\alpha_{\max }}$, neither of which is nilpotent by Lemma 2 . So this cannot occur either and we are done.

Proof of Theorem 1. We complete our proof that $V=\mathfrak{n}$ as follows. We have seen above that we can assume that $V$ is $\mu$-stable and it contains a semi-regular element of the form $x=\sum_{i=1}^{r} x_{i}$ where every $x_{i} \in \mathfrak{g}_{\alpha_{i}}$ is non-zero. Therefore, by Lemma $7, V$ contains all simple root spaces $\mathfrak{g}_{\alpha_{i}}$.

Now let $\lambda: K^{*} \rightarrow T$ be a regular 1-PSG, and put $V_{0}:=\lim _{t \rightarrow 0} \lambda(t) \cdot V$ and $V_{\infty}:=\lim _{t \rightarrow \infty} \lambda(t) \cdot V$. The two limits $V_{0}$ and $V_{\infty}$ are $T$-stable maximal nilpotent subspaces, and both contain all simple root spaces by Lemma 4 (iv). However, then $V_{0}=\mathfrak{n}=V_{\infty}$ by Proposition 1(ii). Hence, $V$ is $\lambda$-stable and thus equal to $\mathfrak{n}$. 


\section{J. Draisma, H. Kraft and J. Kuttler}

\section{Appendix A. Proof of Theorem 1}

The proof of Theorem 1 depends on three ingredients that we will explain in detail here: semi-regular nilpotent elements, the fact that $\left[\mathfrak{g}_{\alpha}, \mathfrak{g}_{-\alpha}\right] \neq 0$, and the validity of Lemma 2 . We continue to use the notation of the main text.

\section{A.1 Semi-regular nilpotent elements}

We call a nilpotent element $x$ of $\mathfrak{g}$ semi-regular if there is exactly one Borel subalgebra of $\mathfrak{g}$ that contains $x$. Regular nilpotent elements are semi-regular, and in sufficiently large characteristic, or in characteristic zero, the converse is also true (cf. [Kos59, Theorem 5.3]).

Lemma A.1. Let $x=\sum_{\alpha \in \Phi^{+}} x_{\alpha}$ with $x_{\alpha} \in \mathfrak{g}_{\alpha}$. Then the following statements are equivalent:

(i) only finitely many Borel subalgebras contain $x$;

(ii) $x_{\alpha_{i}}$ is non-zero for all simple roots $\alpha_{1}, \ldots, \alpha_{r}$; and

(iii) $x$ is semi-regular.

Moreover, the semi-regular nilpotent elements form an open dense set of the nilpotent cone $\mathcal{N}_{\mathfrak{g}}$.

For lack of a suitable reference we include a proof of the lemma.

Proof. To prove that (i) implies (ii), suppose that $x$ is not supported in $\alpha_{1}$, say. Let $P$ be the standard parabolic subgroup corresponding to $\alpha_{1}$ and let $L$ be the Levi subgroup $G_{\alpha_{1}}$ of $P$, so that $L$ is of semi-simple rank 1 . Now $x$ lies in the Lie algebra of the unipotent radical $U_{P}$ of $P$, hence if $B_{L}$ is any Borel subgroup of $L$, then $\operatorname{Lie}\left(B_{L} U_{P}\right)$ is a Borel subalgebra of $\mathfrak{g}$ containing $x$, and the Borel subalgebras thus obtained are all distinct.

To see that (ii) implies (iii) suppose that $x$ is supported in all simple root spaces, and that it lies in $\operatorname{Lie}(B) \cap \operatorname{Lie}\left(B^{\prime}\right)$, where $B^{\prime}$ is a second Borel subgroup of $G$. Now $B \cap B^{\prime}$ contains a maximal torus $T^{\prime}$ of $G$, and it is easy to see that $x$ is also supported in all simple $T^{\prime}$-root spaces. The Borel subalgebras $\operatorname{Lie}(B)$ and $\operatorname{Lie}\left(B^{\prime}\right)$ are both $T^{\prime}$-stable, and hence along with $x$ contain all simple $T^{\prime}$-root spaces. However, then they are equal.

To see that the semi-regular nilpotent elements form an open subset of $\mathcal{N}_{\mathfrak{g}}$ consider the Springer resolution $p: G \times{ }^{B} \mathfrak{n}=(G \times \mathfrak{n}) / B \rightarrow \mathcal{N}_{\mathfrak{g}}$, sending the equivalence class of $(g, x)$ to $g x g^{-1}$. For every $x \in \mathcal{N}_{\mathfrak{g}}$ the fibre $p^{-1}(x)$ is in bijection with the set of Borel subalgebras containing $x$, so $x$ is semiregular if and only if $p^{-1}(x)$ consists of one point. By the above, this is the same as saying that the fibre is finite. Since $p$ is proper, this is an open (and homogeneous) condition on $\mathcal{N}_{\mathfrak{g}}$.

\section{A.2 Almost simple connectedness}

In the proof of Theorem 1 we repeatedly used the fact that $\left[\mathfrak{g}_{\alpha}, \mathfrak{g}_{-\alpha}\right] \neq 0$.

Lemma A.2. If $G$ satisfies Condition 1 then $\left[\mathfrak{g}_{\alpha}, \mathfrak{g}_{-\alpha}\right] \neq 0$ for all roots $\alpha$ of $G$.

Proof. By definition, Lie $G_{\alpha}=\mathfrak{g}_{-\alpha} \oplus \mathfrak{h} \oplus \mathfrak{g}_{\alpha}$ and so the statement is clear if $\left(G_{\alpha}, G_{\alpha}\right) \simeq \mathrm{SL}_{2}$. It also holds if $\left(G_{\alpha}, G_{\alpha}\right) \simeq \mathrm{PSL}_{2}$ and char $K \neq 2$, because then the morphism $\mathrm{SL}_{2} \rightarrow \mathrm{PSL}_{2}$ is separable and thus induces an isomorphism on the Lie algebras.

The following lemma exhibits a sufficient condition for Conditions 1 and 2 to hold. Recall that $G$ is said to be almost simply connected if all simple components of $(G, G)$ are simply connected. 


\section{MAXimal NiLPotent SUBSPACES}

Lemma A.3. Suppose that $G$ is almost simply connected. Then the same is true for every Levi subgroup $G_{\Psi} \subset G$, where $\Psi$ is a subsystem of $\Phi$. In particular, this implies Conditions 1 and 2.

Proof. Let $\Psi$ be a subsystem of the root system $\Phi$ of $(G, T)$ and consider the corresponding Levi subgroup $L:=G_{\Psi}$. To show that every simple component of $(L, L)$ is simply connected, we may as well assume that $\Psi$ itself is already simple. Replacing $G$ by the simple component of $(G, G)$ whose root system contains $\Psi$, we may also assume that $G$ is simple, and hence simply connected by assumption.

Choose a system of simple roots $\alpha_{1}, \ldots, \alpha_{r}$ in $\Phi$ containing a system of simple roots of $\Psi$. For every $\alpha_{i}$, let $\check{\alpha}_{i}$ be the coroot of $\alpha_{i}$, i.e. the unique element of $Y(T)_{\mathbb{R}}$ that satisfies

$$
r_{\alpha_{i}}(x)=x-\left\langle x, \check{\alpha}_{i}\right\rangle \alpha_{i} \text { for all } x \in X(T),
$$

where $r_{\alpha_{i}}$ is the reflection of $X(T)$ generating the Weyl group of $\left(G_{\alpha_{i}}, T\right)$ (see [Spr98, Lemma 7.1.8]). Denote by $T_{\alpha_{i}}$ the maximal torus of $\left(G_{\alpha_{i}}, G_{\alpha_{i}}\right)$ contained in $T$; then $\check{\alpha}_{i}$ actually lies in $Y\left(T_{\alpha_{i}}\right) \subseteq Y(T)$ (see [Spr98, Lemma 7.3.5]), i.e. it is a 1-PSG into $T_{\alpha_{i}}$. The simple connectedness of $G$ means by definition that the lattice

$$
P:=\left\{x \in X(T)_{\mathbb{R}} \mid\left\langle x, \check{\alpha}_{i}\right\rangle \in \mathbb{Z} \text { for all } i=1, \ldots, r\right\},
$$

which clearly contains $X(T)$, is actually equal to $X(T)$ (see [Spr98, 8.1.11]). This is equivalent to the condition that the basis $\left\{\omega_{i}\right\}_{i}$ of $X(T)_{\mathbb{R}}$ dual to $\left\{\check{\alpha}_{i}\right\}_{i}$, consisting of the fundamental weights, lies in the lattice $X(T)$.

Now let $T_{L}$ be the maximal torus of $(L, L)$ contained in $T$. The coroots of those $\alpha_{i}$ that lie in $\Psi$ are the same $\check{\alpha}_{i}$ as before. To see that the lattice

$$
P_{L}:=\left\{x \in X\left(T_{L}\right)_{\mathbb{R}} \mid\left\langle x, \check{\alpha}_{i}\right\rangle \in \mathbb{Z} \text { for all } \alpha_{i} \in \Psi\right\}
$$

equals $X\left(T_{L}\right)$, we need only observe that the restrictions to $T_{L}$ of the $\omega_{i} \in X(T)$ with $\alpha_{i} \in \Psi$ are the fundamental weights for $(L, L)$. Hence $(L, L)$ is simply connected.

\section{A.3 Anomalies}

We give some constructions of nilpotent subspaces that are not contained in a maximal nilpotent subalgebra. Most of them are in fact $T$-stable, in which case the following lemma shows that they are not conjugate to $\mathfrak{n}$.

Lemma A.4. Suppose that $V$ is a $T$-stable nilpotent subspace of $\mathfrak{g}$ that is $G$-conjugate to $\mathfrak{n}$. Then $V$ is in fact $W$-conjugate to $\mathfrak{n}$.

As the stabilizer of $\mathfrak{n}$ in $G$ is $B$, this lemma follows from the fact that the $T$-fixed points in $G / B$ form one $W$-orbit [Bor91, Proposition 11.19]. For the reader's convenience we include a short direct proof.

Proof. Suppose that $g \in G$ conjugates $\mathfrak{n}$ into $V$, i.e. that $g \mathfrak{n}=V$. By the Bruhat decomposition we have $g=b^{-1} w b^{\prime}$, where $b, b^{\prime} \in B$ and where $w$ represents an element of $W$. As $\mathfrak{n}$ is $b^{\prime}$-stable, we find $w \mathfrak{n}=b V$. We claim that $b V=V$. First, $V$ is $T$-stable by assumption and $b V$ is $T$-stable because $b V=w \mathfrak{n}$. Now let $x_{\alpha} \in V \cap \mathfrak{g}_{\alpha}$ be a root vector. Then $b x_{\alpha}$ is a non-zero multiple of $x_{\alpha}$ plus root vectors corresponding to roots higher than $\alpha$, and by $T$-stability of $b V$ the non-zero component of $b x_{\alpha}$ in $\mathfrak{g}_{\alpha}$ lies in $b V$. Hence $V \subseteq b V$ and therefore $w \mathfrak{n}=b V=V$.

Example A.1. Suppose that char $K=0$ and that $\mathfrak{g}$ is simple of rank 2 with simple roots $\alpha_{1}, \alpha_{2}$, where $\alpha_{1}$ is short. 


\section{J. Draisma, H. Kraft and J. Kuttler}

(i) If $\mathfrak{g}$ is of type $C_{2}$, then the linear subspace $V:=\mathfrak{g}_{\alpha_{1}} \oplus \mathfrak{g}_{\alpha_{2}} \oplus \mathfrak{g}_{-\alpha_{1}-\alpha_{2}}$ of dimension three consists of nilpotent elements. In fact, it corresponds to the matrices in $\mathfrak{s p}_{4}$ of the form

$$
x:=\left[\begin{array}{cccc}
0 & r & 0 & 0 \\
0 & 0 & s & 0 \\
t & 0 & 0 & -r \\
0 & t & 0 & 0
\end{array}\right] \quad(r, s, t \in K),
$$

where we use the embedding $\mathrm{Sp}_{4} \subset \mathrm{GL}_{4}$ defined by the 'skew diagonal form' $(x, y):=x_{1} y_{4}+$ $x_{2} y_{3}-x_{3} y_{2}-x_{4} y_{1}$; and raising $x$ to the fourth power yields zero. On the other hand, $V$ is not conjugate to a subspace of $\mathfrak{n}$ by Lemma A.4.

(ii) Similarly, for $\mathfrak{g}$ of type $G_{2}$ any element of the five-dimensional subspace $V:=\mathfrak{g}_{\alpha_{1}} \oplus \mathfrak{g}_{\alpha_{2}} \oplus$ $\mathfrak{g}_{\alpha_{1}+\alpha_{2}} \oplus \mathfrak{g}_{-2 \alpha_{1}-\alpha_{2}} \oplus \mathfrak{g}_{-3 \alpha_{1}-\alpha_{2}}$ has a matrix of the form

$$
\left[\begin{array}{ccccccc}
0 & r & t & 0 & 0 & 0 & 0 \\
0 & 0 & -s & -2 t & 0 & 0 & 0 \\
0 & 0 & 0 & 2 r & 0 & 0 & 0 \\
u & 0 & 0 & 0 & r & -t & 0 \\
v & -u & 0 & 0 & 0 & s & t \\
0 & 0 & u & 0 & 0 & 0 & r \\
0 & 0 & v & -2 u & 0 & 0 & 0
\end{array}\right]
$$

relative to a weight basis of the seven-dimensional $\mathfrak{g}$-module. A direct computation shows that the seventh power of this matrix is zero. Hence $V$ is nilpotent, but it is not conjugate to a subspace of $\mathfrak{n}$ by Lemma A.4.

(iii) In $\mathfrak{g}$ of type $A_{2}$ there are no $T$-stable nilpotent subspaces that are not conjugate to a subspace of $\mathfrak{n}$. However, the subspace

$$
\left\{\left[\begin{array}{ccc}
0 & s & 0 \\
t & 0 & s \\
0 & -t & 0
\end{array}\right] \mid s, t \in K\right\}
$$

is nilpotent, but generates $\mathfrak{g}$ as a Lie algebra.

Next we recall the exceptions leading to our Conditions 1-3. In each case Lemma A.4 shows that the constructed subspaces are not $G$-conjugate to $\mathfrak{n}$.

Example A.2.

(i) As explained in $\S 1$, the subspace $\mathfrak{g}_{-\alpha} \oplus \mathfrak{g}_{\alpha}$ of $\mathfrak{g}=\mathfrak{p s l}_{2}$ is nilpotent in characteristic two, although of dimension larger than $1=\operatorname{dim} \mathfrak{n}$.

(ii) Similarly, the subspace $\mathfrak{g}_{\alpha_{1}} \oplus \mathfrak{g}_{\alpha_{2}} \oplus \mathfrak{g}_{-\alpha_{1}-\alpha_{2}}$ of $\mathfrak{p s t}_{3}$ is nilpotent in characteristic three, and not conjugate to $\mathfrak{n}$ while of dimension $3=\operatorname{dim} \mathfrak{n}$.

(iii) The same argument shows that the subspace $V:=\mathfrak{g}_{\alpha_{1}} \oplus \mathfrak{g}_{\alpha_{2}} \oplus \mathfrak{g}_{\alpha_{1}+\alpha_{2}} \oplus \mathfrak{g}_{-2 \alpha_{1}-\alpha_{2}}$ in $\mathfrak{p s p}_{4}$ is nilpotent in characteristic two. In fact, $V$ is the image of the subspace

$$
U:=\left\{\left[\begin{array}{cccc}
0 & r & t & 0 \\
0 & 0 & s & t \\
0 & 0 & 0 & -r \\
u & 0 & 0 & 0
\end{array}\right] \mid r, s, t, u \in K\right\} \subset \mathfrak{s p}_{4}
$$

under the adjoint representation. A short calculation shows that $A^{4}$ is a scalar matrix for all $A \in U$ which implies, in characteristic two, that the image of $A$ in $\mathfrak{p s p}_{4}$ is nilpotent. Hence, $V$ is nilpotent of dimension $4=\operatorname{dim} \mathfrak{n}$, but $V$ is not conjugate to $\mathfrak{n}$. This example is 


\section{MAXimal NiLPotent SUBSPACES}

excluded from our Theorem 1 because of Condition 1: for all root subgroups $G_{\alpha} \subset \mathrm{PSp}_{4}$ one has $\left(G_{\alpha}, G_{\alpha}\right) \simeq \mathrm{PSL}_{2}$.

(iv) If char $K=2$ and $G$ is of type $G_{2}$, then the space

$$
V:=\mathfrak{g}_{-2 \alpha_{1}-\alpha_{2}} \oplus \bigoplus_{\alpha \in \Phi^{+} \backslash\left\{2 \alpha_{1}+\alpha_{2}\right\}} \mathfrak{g}_{\alpha}
$$

is a six-dimensional $T$-stable nilpotent subspace of $\mathfrak{g}$, but not conjugate to a maximal nilpotent subalgebra of $\mathfrak{g}$ (see Example 2 in $\S 1$ ).

\section{A.4 Proof of Lemma 2}

We write $\alpha, \beta$ for the simple roots, where $\alpha$ is short, and $\alpha_{\max }$ for the highest root. Unfortunately, the proof needs a case-by-case analysis.

The case $A_{2}$. If char $K$ is not 3 , then $\mathfrak{g}$ is isomorphic to $\mathfrak{s l}_{3}$, with the adjoint representation factoring through $\mathrm{PGL}_{3}$. If char $K=3$, then $G$ is isomorphic to $\mathrm{SL}_{3}$ by Condition 2 .

In either case, it suffices to treat the case where $G=\mathrm{SL}_{3}$. Now in the defining representation of $\mathrm{SL}_{3}$, where we choose $B$ and $T$ as usual, any matrix supported in $\mathfrak{g}_{\alpha}, \mathfrak{g}_{\beta}$, and $\mathfrak{g}_{-\alpha_{\max }}$ has a non-zero determinant and is therefore not nilpotent.

The case $C_{2}$. If char $K \neq 2$, then $\mathfrak{g}$ is isomorphic to $\mathfrak{s p}_{4}$, with the adjoint representation factoring through $\mathrm{PSp}_{4}$. If char $K=2$, then Condition 1 assures that $G$ is isomorphic to $\mathrm{Sp}_{4}$, as the subgroup $\left(G_{\alpha}, G_{\alpha}\right)$ in $\mathrm{PSp}_{4}$ is isomorphic to $\mathrm{PSL}_{2}$ (see Example A.2(iii) above).

Hence we need only deal with the case where $G=\mathrm{Sp}_{4}$, defined as above by the 'skew diagonal form' $(x, y):=x_{1} y_{4}+x_{2} y_{3}-x_{3} y_{2}-x_{4} y_{1}$. With $T$ consisting of diagonal matrices and $B$ of upper triangular matrices, any element $x$ of $\mathfrak{s p}_{4}$ supported exactly in $\left\{\alpha, \beta,-\alpha_{\max }\right\}$ is of the form

$$
x=\left[\begin{array}{cccc}
0 & r & 0 & 0 \\
0 & 0 & s & 0 \\
0 & 0 & 0 & -r \\
t & 0 & 0 & 0
\end{array}\right]
$$

with $r, s, t \neq 0$. However, then $\operatorname{det} x \neq 0$, hence $x$ is not nilpotent.

The case $G_{2}$. This is a variation of the argument for $C_{2}$ and $A_{2}$. Recall that char $K \neq 2$ by Condition 2. We will use the fact that the two-dimensional and the three-dimensional representations of $\mathrm{SL}_{2}$ of highest weight 1 and 2, respectively, remain irreducible in char $K \neq 2$, and that $\mathfrak{s l}_{2}$ acts as usual on them.

Recall that $G$ of type $G_{2}$ is simply connected. Let $V$ be its seven-dimensional representation corresponding to the fundamental weight $2 \alpha+\beta$, and write $V_{\gamma}$ for the $\gamma$-weight space of $V$. Let $\delta$ be a root and let $\gamma$ be a weight in $V$ for which $\gamma+\delta$ is a weight in $V$. Then the observation above applied to $G_{\delta} \cong \mathrm{SL}_{2}$ shows that $x_{\delta} V_{\gamma} \neq 0$ for all non-zero $x_{\delta} \in \mathfrak{g}_{\delta}$.

Now let $x=x_{\alpha}+x_{\beta}+x_{-\alpha_{\max }}$ be an element of $\mathfrak{g}$ with $x_{\alpha} \in \mathfrak{g}_{\alpha}, x_{\beta} \in \mathfrak{g}_{\beta}$, and $x_{-\alpha_{\max }} \in \mathfrak{g}_{-\alpha_{\max }}$ all non-zero. Then one readily verifies that $x^{5} V_{2 \alpha+\beta}=V_{\alpha+\beta}$. It therefore is enough to show that $x^{2} V_{\alpha+\beta} \neq 0$. Let $v \in V_{\alpha+\beta}$ be non-zero. Now compute

$$
x^{2} v=x\left(x_{\alpha} v+x_{-\alpha_{\max }} v\right)=x_{-\alpha_{\max }} x_{\alpha} v+x_{\alpha} x_{-\alpha_{\max }} v ;
$$

as $-\alpha_{\max }+\alpha$ is not a root, the corresponding components of $x$ commute and the last expression is equal to $2 x_{\alpha} x_{-\alpha_{\max }} v$ and thus non-zero, as claimed.

This finishes the proof of Lemma 2. 


\section{MAXimal Nilpotent SUbSPACES}

\section{ACKNOWLEDGEMENT}

The third author would like to thank Northeastern University in Boston for its kind hospitality during much of his work on this paper.

\section{REFERENCES}

Bor91 A. Borel, Linear algebraic groups, second enlarged edition, Graduate Texts in Mathematics, vol. 126 (Springer, Berlin, 1991).

Bou68 N. Bourbaki, Groupes et algèbres de Lie, Éléments de mathématiques (Hermann, 1968), ch. 4-6.

CRT98 A. Causa, R. Re and T. Teodorescu, Some remarks on linear spaces of nilpotent matrices, Matematiche (Catania) 53 (Suppl.) (1998), 23-32.

Fas97 M. A. Fasoli, Classification of nilpotent linear spaces in $M(4 ; \mathbb{C})$, Comm. Algebra 25 (1997), 1919-1932.

FP96 M. A. Fasoli and F. Pauer, On certain linear spaces of nilpotent matrices, Comm. Algebra 24 (1996), 3149-3154.

Ger58 M. Gerstenhaber, On nilalgebras and linear varieties of nilpotent matrices, I, Amer. J. Math. 80 (1958), 614-622.

Ger59a M. Gerstenhaber, On nilalgebras and linear varieties of nilpotent matrices, II, Duke Math. J. 27 (1959), 21-31.

Ger59b M. Gerstenhaber, On nilalgebras and linear varieties of nilpotent matrices, III, Ann. of Math. (2) 70 (1959), 167-205.

Ger62 M. Gerstenhaber, On nilalgebras and linear varieties of nilpotent matrices, IV, Ann. of Math. (2) 75 (1962), 382-418.

Hum75 J. E. Humphreys, Linear algebraic groups (Springer, Berlin, 1975).

Jan03 J. C. Jantzen, Representations of algebraic groups, Mathematical Surveys and Monographs, vol. 107 (American Mathematical Society, Providence, RI, 2003).

Kal73 W. L. J. van der Kallen, Infinitesimally central extensions of Chevalley groups, Lecture Notes in Mathematics, vol. 356 (Springer, Berlin, 1973).

KKL89 F. Knop, H. Kraft, D. Luna and Th. Vust, Local properties of algebraic group actions, in Algebraische Transformationsgruppen und Invariantentheorie, eds H. Kraft, P. Slodowy and T. A. Springer, DMV-Seminar, vol. 13 (Birkhäuser, Basel, 1989), 63-75.

Kos59 B. Kostant, The principal three-dimensional subgroup and the Betti numbers of a complex simple Lie group, Amer. J. Math. 81 (1959), 973-1032.

MOR91 B. Mathes, M. Omladic and H. Radjavi, Linear spaces of nilpotent matrices, Linear Algebra Appl. 149 (1991), 215-225.

MR98 R. Meshulam and N. Radwan, On linear subspaces of nilpotent elements in a Lie algebra, Linear Algebra Appl. 279 (1998), 195-199.

Spr98 T. A. Springer, Linear algebraic groups, Progress in Mathematics, vol. 9, second edition (Birkhäuser, Basel, 1998).

SV00 T. A. Springer and F. D. Veldkamp, Octonions, Jordan algebras and exceptional groups, Springer Monographs in Mathematics (Springer, Berlin, 2000).

Sum74 H. Sumihiro, Equivariant completion, J. Math. Kyoto Univ. 14 (1974) 1-28.

Sum75 H. Sumihiro, Equivariant completion, II, J. Math. Kyoto Univ. 15 (1975) 573-605.

Jan Draisma jan.draisma@unibas.ch

Mathematisches Institut, Universität Basel, Rheinsprung 21, CH-4051 Basel, Switzerland

Hanspeter Kraft hanspeter.kraft@unibas.ch

Mathematisches Institut, Universität Basel, Rheinsprung 21, CH-4051 Basel, Switzerland

Jochen Kuttler jochen.kuttler@web.de

Department of Mathematics, Northeastern University, 360 Huntington Avenue, Boston MA 02115, USA 